\title{
SMALL SCALE LANDFORM MAPPING BY INTEGRATED OPTICAL (2D) AND TERRAIN (3D) UAV DATA
}

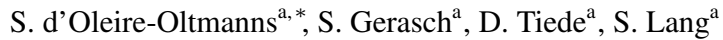 \\ ${ }^{\text {a }}$ Department of Geoinformatics - Z_GIS, University of Salzburg \\ Schillerstr. 5020 Salzburg, Austria - sebastian.doleire-oltmanns@sbg.ac.at
}

KEY WORDS: landform mapping, UAV, monitoring, local scale, ditch

\begin{abstract}
:
The potential of UAV-based data for fine-scaled remote sensing applications has been investigated for several years and is still increasing. The presented study is attached to the field of landform mapping. Mapping and extracting of landforms contributes to different sectors such as agriculture, disaster management and nature conservation. In this study recent experiences and results from working in a nature conservation area are presented. The chosen study area 'Haarmoos' is located southwest of the lake Abtsdorf, Bavaria and is declared under the European wide Natura 2000 network. Optical imagery and terrain data were analyzed in an integrated approach based on object-based image analysis (OBIA). The classification delivered good results. Ditches may be classified well by the presented approach whereas a tradeoff was made between local rule set adaption and applicability to further data sets. The validation of the classification results was based on three steps.
\end{abstract}

\section{INTRODUCTION}

The potential of UAV-based data for fine-scaled remote sensing applications has been investigated for several years and is still increasing. This is due to optical sensors with even higher spatial resolution as well as new type of sensors that may be carried on UAVs. In the field of landform mapping the use of UAVs has recently started to become more attractive for local scale applications and is increasingly used for photogrammetric and remote sensing applications (Colomina and Molina, 2014). Mapping and extracting of landforms contributes to different sectors such as agriculture, disaster management and nature conservation. This study presents recent experiences and results in dealing with landform mapping on small scales in a conservation area based on optical (2D) and derived terrain (3D) data acquired from UAV missions. Object-based image analysis (OBIA) has already been applied in several studies on landform mapping and supports a strategy of integrated data analysis across different scales (Anders et al., 2011, D’Oleire-Oltmanns et al., 2013).

\section{METHODOLOGY}

\subsection{Study area}

The chosen study area Haarmoos is located southwest of the lake Abtsdorf, south of the city of Laufen, Bavaria (see figure 1). The area is a historically drained wetland site, a fen which is nowadays protected by different nature conservation acts: the site is declared under the European wide Natura 2000 network and adheres to federal regulations of bird protection as a unique habitat for certain wet meadow breading birds in Bavaria. As these wet meadows requires a distinct amount of water constantly available, the maintenance and even extension of drainage ditches is considered as potential risk to this sensitive environment. The application of UAV-based remote sensing in combination with e.g. VHR optical satellite data as well as in-situ data offers a valuable approach for the mapping and monitoring of such sensitive and partly inaccessible habitats.

\footnotetext{
${ }^{*}$ Corresponding author
}

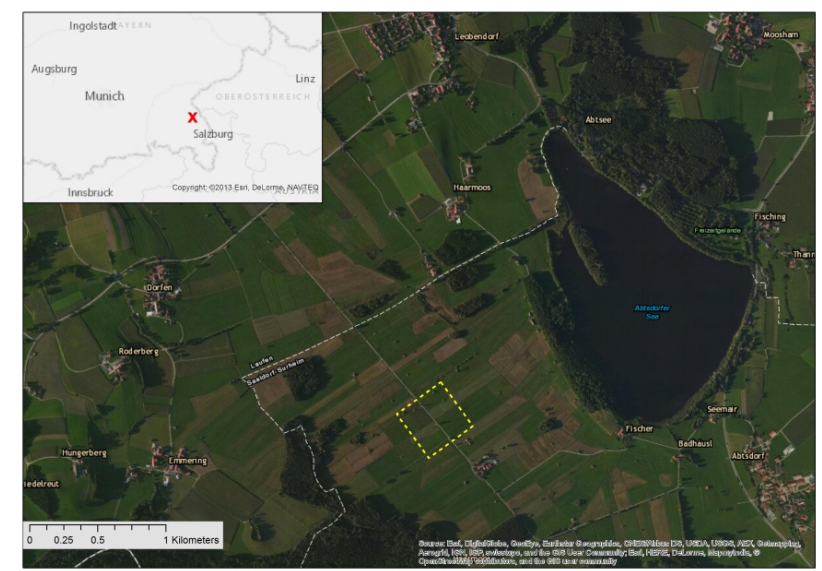

Figure 1. Study area located south-west of the lake Abtsdorf. The dashed yellow line illustrates the extent of the mapped area during the UAV campaign.

In order to provide a more realistic impression of the study area some oblique aerial photographs have been acquired. In figure 2 the road parallel to the main ditch may be identified from left to right in the middle of the photograph. Furthermore the structure of the area characterized by field plots and ditches in alternating order is visible.

\subsection{Data acquisition and photogrammetric processing}

The data acquisition has been conducted with a multicopter that is equipped with a consumer-grade RGB camera (model RICOH GXR). Flight campaigns followed pre-defined flightpaths that met all requirements for future photogrammetric processing of the aerial photographs: in-track and parallel overlap of both $70 \%$ and a flying height of $100 \mathrm{~m}$ above ground ensuring a Ground Sample Distance (GSD) below $5 \mathrm{~cm}$ per pixel.

Distributed Ground Control Points (GCPs) were measured with a dGPS to increase the accuracy during the triangulation of the aerial photographs and the calculation of data products such as 


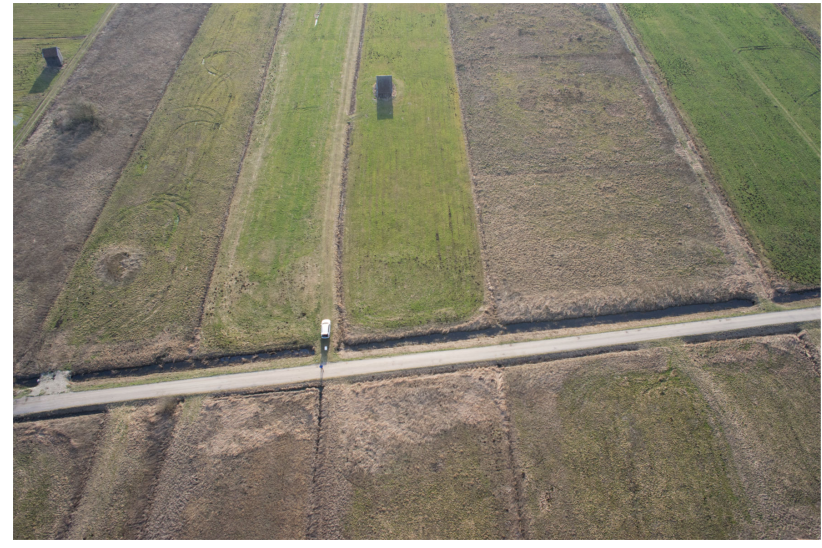

Figure 2. Oblique aerial photograph providing a more detailed view on the study area. The ditches between the field plots may be easily identified. In the center area the UAV pilot and the parked car may be found.

orthomosaics and Digital Surface Models (DSMs). In figure 3 the distribuion of the GCPs is illustrated.

\section{Ground Control Points}

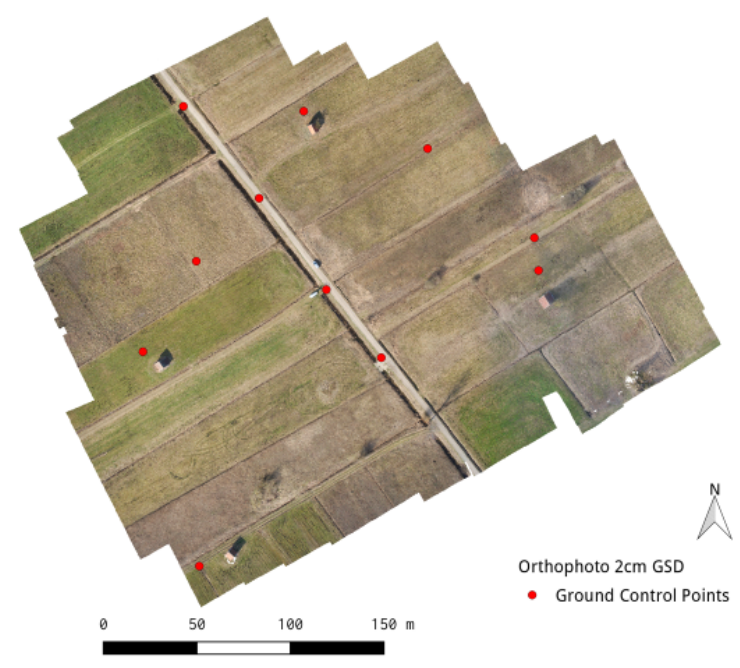

Figure 3. Distribution of GCPs (red points) over the mapped area.

Two flights were conducted to cover the chosen study site. The acquired aerial photographs were processed by using a software environment (Agisoft PhotoScan) that is based on the Structurefrom-Motion (SfM) algorithm. The acquired raw images were triangulated based on the software implicit feature matching routines. Orientation of the image block in WGS84 took place based on the measured reference coordinates. The measured GCP coordinates were assigned to the corresponding image pixel containing the GCP. This allows to reference the image block accurately with a much higher precision than relying only on the accuracy of the onboard GPS values.

After referencing and optimization of the triangulation several data products were calculated: A point-cloud representing the three dimensional surface of the mapped study site was generated. Based on this point cloud a mesh data set was derived that builds the basis for the calculation of the Digital Surface Model
(DSM). The DSM had a resolution of $2 \mathrm{~cm}$ per pixel which represents the mapped study site on a very high level of detail.

\subsection{Classification of ditches and validation}

Preprocessing. The resulting elevation model had an original resolution of $2 \mathrm{~cm}$ per pixel. Since the project's scope lies on the landform as such and not on distinct vegetation features or single trunks, the elevation model was smoothed using a moving window with a size of $15 \times 15$ pixel. This step preserved the general landform needed for the analysis and reduced the amount of influence of single vegetation features. In addition, a slope layer was derived from the elevation model according to (Mitasova and Hofierka, 1993). The size of one image layer was around 2 GB. To speed up the following rule-set development in CNL (Cognition Network Language within the eCognition software, Trimble Geospatial), we decided to choose a subset of the data with a size of approximately $0.25 \mathrm{ha}$, selecting a heterogeneous area in order to develop a rule-set which would also be applicable for the whole dataset (see figure 3).

OBIA. At an initial step, the height model and the slope were inspected visually. For a human expert, it was already possible to differentiate the ditches from other terrain forms (see figure 3 ). Based on these observations the goal was to develop a classification rule-set that mimics the human interpreter best possible. But also provides an easy to use and easy to understand framework for further adaption to other datasets. The following steps were conducted:

1. Initial segmentation based on the Multiresolution Segmentation algorithm by (Baatz and Schäpe, 2000). The aim was to derive homogeneous objects in respect to the two parameters slope and elevation (input layers for the segmentation process, the RGB images were not used). The ESP tool from Dragut et al. (Dragut et al., 2010) was used to automate the process of parameter selection and achieve a result with maximized local variance between the image segments.

2. Resulting objects were classified as ditch objects when the slope threshold (manually defined) exceeded 14 degrees. Falsely classified objects (non ditch) with high slope values were removed from the classification through excluding objects by relative local height values. The form descriptor length to width ratio was used to differentiate between ditch objects and non-elongated object with similar slope values. As a final step, objects with lower slope values surrounded by the objects of the class ditch (ditch bottoms) were also classified as ditch. Only 9 different rules were needed for the ditch extraction routine, which was possible due to the clear distinction of ditches in the data set (elongated structures showing local minimum height values in the centre and high slopes values at the border), keeping therefore the complexity of the rule-set low and allow an easier transferability to other areas.

Validation. Three steps were taken in order to validate the results from the classification approach. In the first step, we compared the classification result to the RGB orthophoto. With the knowledge of the field campaign, we were able to identify the position of the ditches. This first indication was used to further improve and change the algorithm until the presented state. As a second step, the classified ditches were assessed against a manually delineated reference data set. This dataset was created by an expert independently of and before the automatized delineation (see figure 4). 

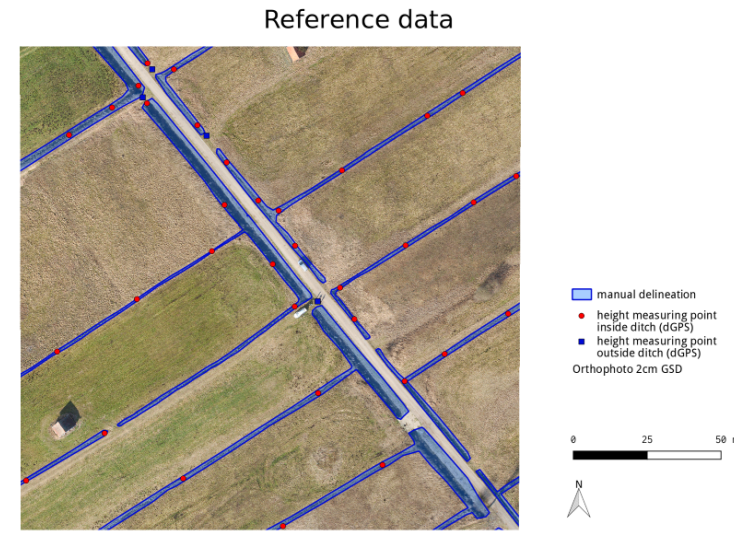

Figure 4. Manually delineated reference data (blue polygons) and the distribution of height measuring points are illustrated. Points are colored according to their location inside (red) or outside(blue) the ditch.

In a third step, the classification was compared with ground truth data that was acquired by a second survey (see figure 4). For this purpose, we captured the height of 40 different locations with a differential GPS. Most heights where located directly in the ditches (red circles) and some additional heights were captured between the ditches (blue rectangles) on the small bridges between the meadow sites. In addition to the evaluation of the $2 \mathrm{D}$ position, the heights of the points were compared to buffered elevation model areas with a radius of $20 \mathrm{~cm}$.

\section{RESULTS AND DISCUSSION}

The first look onto the result reveals a good classification result. The protruding lines forming the ditches are clearly visible in the classification, too. On a closer look, the classification was found to over classify the main ditch. This was also detected when the classification was compared to the expert segmentation of the ditches (see underlying classification result in figure 5).

\section{Rule set based classification result}

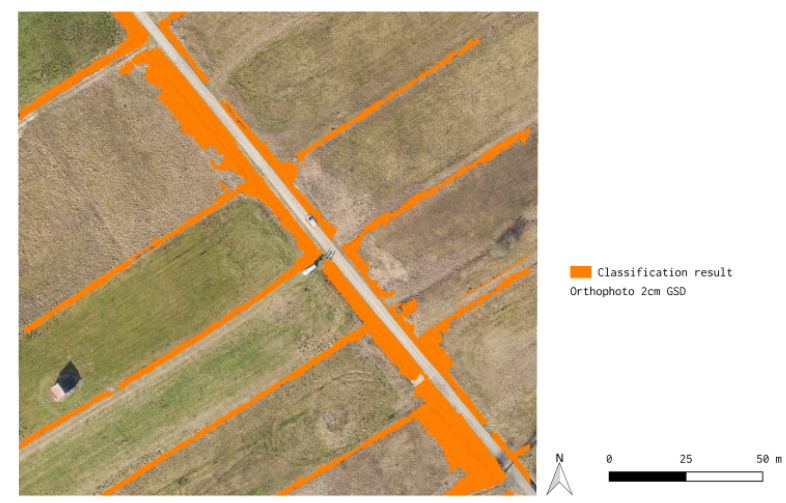

Figure 5. Classification Results (orange). Some overclassification along the main ditch is visible. The overall classification result is showing valid outcomes.

In figure 6 the classification results and the reference data is illustrated for validation purpose. The good classification outcome is illustrated more detailed in figure 7 showing a subset of the overall area that was analyzed.

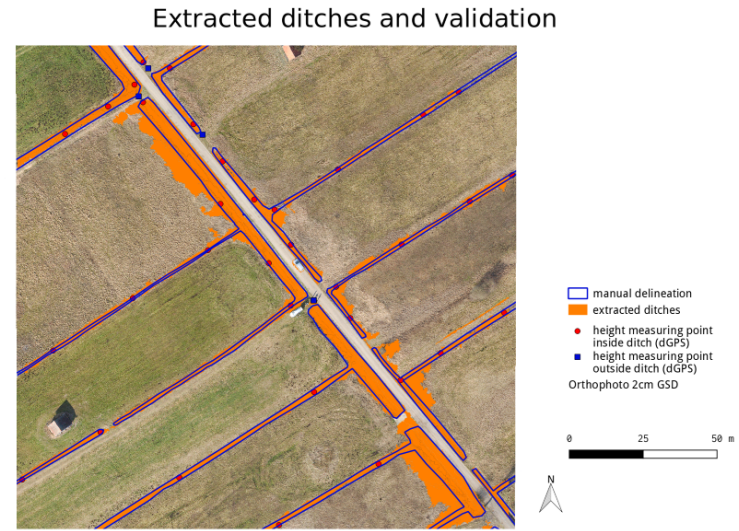

Figure 6. Illustration of the classification outcomes (orange) and the reference data (blue outlines) including the height measuring points.

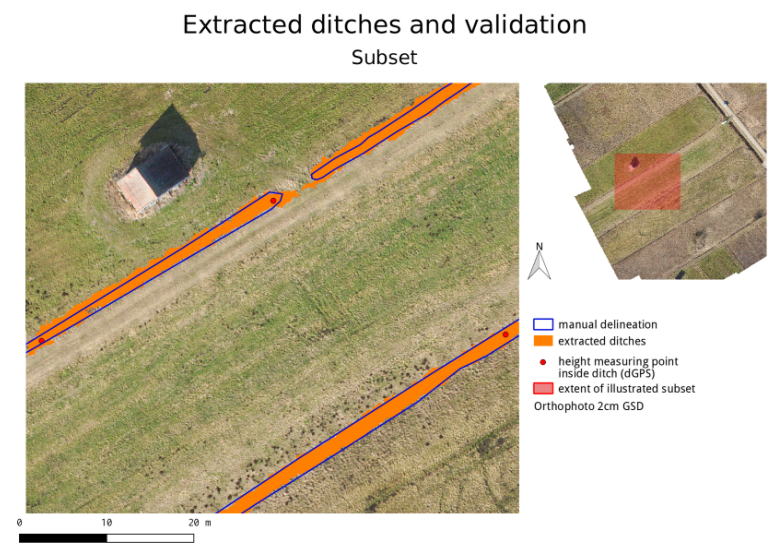

Figure 7. A subset of the classification outcomes (orange) and the reference data (blue outlines). The location of the subset is indicated by the red rectangular in the overview.

In comparison to the ground truth data, all but one point were correctly classified as ditches. The height difference between the points and the elevation model was found to be significant. The mean error is located at $0.5 \mathrm{~m}+/-0.41 \mathrm{~m}$ (see figure 8) This error can be explained by the fact, that the ground of the ditches was measured, but at the time of the UAS flight, some ditches were filled with water partially. Another important factor is the vegetation to be found within the ditches as well as vegetation which grows over the ditches. These impacts the calculation of the elevation model from the SfM data.

Because of the chosen methodology, the high slope value was used as classification criterion mainly. Therefore the rule set tends to classify rough surfaces such as high grass as ditch. This can be observed by an overclassification of the main drainage ditch along the road. Further rules were tested on the subset as well as on the large scale, but were mostly found to train the rule set too well for the given subset. A tradeoff was made between local rule set adaption and applicability to other data sets.

\section{CONCLUSION}

Mapping and extracting of landforms is a continuous topic contributing to different sectors such as agriculture, disaster manage- 


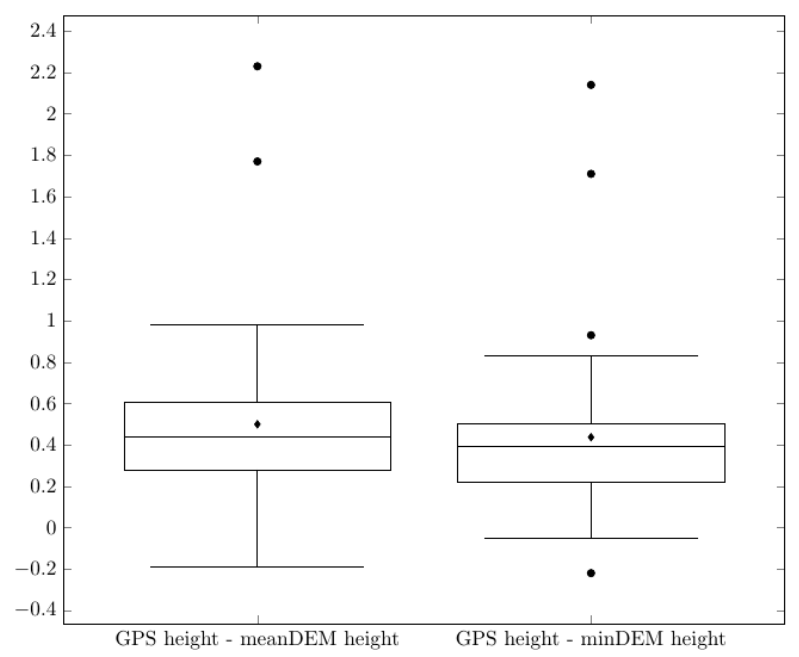

Figure 8. Boxplots illustrating the validation outcomes between GPS height and mean DEM height (left) and GPS height and min DEM height (right).

ment and nature conservation. This study presents recent experiences and results in coping with landform mapping on small scales in a conservation area based on optical (2D) and derived terrain (3D) data acquired from UAV missions. The resulting orthoimage mosaic as well as the elevation model has been analyzed by means of the OBIA concept. The elevation model had an original resolution of $2 \mathrm{~cm}$ per pixel that was smoothed over an area of $30 \mathrm{~cm}$, which preserved the general landform required for the analysis and reduced the amount of influence of single vegetation features. Automated extraction of ditches was conducted based on a clear and simple rule-set, which was possible due to the clear distinction of the ditches in the data sets. A threefold validation approach has been applied for evaluation of the classification results. The height difference between the points and the elevation model was found to be significant. The mean error is located at $0.5 \mathrm{~m}+/-0.41 \mathrm{~m}$ and can be explained by the fact, that the ground of the ditches was measured, but at the time of the UAS flight, some ditches were filled with water partially.

\section{REFERENCES}

Anders, N. S., Seijmonsbergen, A. C. and Bouten, W., 2011. Segmentation optimization and stratified object-based analysis for semi-automated geomorphological mapping. Remote Sensing of Environment 115(12), pp. 2976-2985.

Baatz, M. and Schäpe, A., 2000. Multiresolution segmentation: an optimization approach for high quality multi-scale image segmentation.

Colomina, I. and Molina, P., 2014. Unmanned aerial systems for photogrammetry and remote sensing: A review. ISPRS Journal of Photogrammetry and Remote Sensing 92, pp. 79-97.

D’Oleire-Oltmanns, S., Eisank, C., Dragut, L. and Blaschke, T., 2013. An Object-Based Workflow to Extract Landforms at Multiple Scales From Two Distinct Data Types. IEEE Geoscience and Remote Sensing Letters 10(4), pp. 947-951.

Dragut, L., Tiede, D. and Levick, S. R., 2010. Esp: a tool to estimate scale parameter for multiresolution image segmentation of remotely sensed data. International Journal of Geographical Information Science 24(6), pp. 859-871.
Mitasova, H. and Hofierka, J., 1993. Interpolation by regularized spline with tension. ii: Application to terrain modeling and surface geometry analysis. Mathematical Geology 25(6), pp. 657669. 